\title{
Oman Urological Society Conference 2019
}

Sultan Qaboos University, Muscat, Oman, 3-5 October 2019

\section{مؤتمر الجمعية العمانية لجراحة المسالك البولية 2019}

جامعة السلطان قابوس، مسقط، عمان، 3-5 أكتوبر 2019

\section{Poster Presentations}

\author{
Investigation of Effects of Metformin in Prevention of Bladder Cancer Recurrence \\ Alireza Ghadian \\ Baqiyatallah University of Medical Sciences, Tehran, Iran. E-mail: P_Ghadian@yahoo.com
}

Objectives: First lines of evidence have shown that metformin inhibits cancer cell growth and prevents cancer occurrence in patients with type 2 diabetes. This study aimed to investigate the hypothesised inhibitory effects of metformin on recurrence of bladder cancer in patients undergoing trans-urethral resection of bladder tumour (TURT). Methods: This case-controlled clinical trial included 65 patients who were divided into two groups: a metformin group $(n=32)$ and a control group $(n=33)$. Patients in the metformin group received two tablets of metformin $(500 \mathrm{mg}$ ) daily for one year. Descriptive, comparative and inferential tests were used to compare the frequency of tumour recurrence in terms of gender, grade and stage of the previous tumour among the two groups. Results: There was no significant difference in frequency of tumour recurrence between the metformin and control groups $(P>0.05)$. Although time of tumour recurrence in the metformin group was higher compared to the control group, this increase was not statistically significant $(P$ $>0.05$ ). In addition, tumour recurrence had no correlation with gender and grade of tumour. However, an insignificant positive weak correlation was observed between recurrence and stage of tumour only in the control group $(P>0.05)$. Conclusion: These findings indicate that metformin has no considerable inhibitory effect on recurrence of bladder cancer but may delay tumour recurrence. However, further studies with larger sample sizes and the use of higher doses of metformin for a longer duration of time is suggested to confirm these results.

\section{The Incidence of Bacteriuria after Cystoscopy in Patients with History of Bladder Tumour \\ Alireza Ghadian \\ Baqiyatallah University of Medical Sciences, Tehran, Iran. E-mail: P_Ghadian@yahoo.com}

Objectives: Bladder cancer is the eighth most common cause of death from cancers and is responsible for $3 \%$ of all tumours. Several diagnostic treatments have been proposed for bladder cancer, one of which is flexible cystoscopy. This study aimed to evaluate asymptomatic and symptomatic bacteriuria in patients undergoing cystoscopy for bladder cancer. Methods: This descriptive study included patients who needed cystoscopy for bladder cancer and had been referred to Baghiyyatollah al-Azam Military Hospital, Tehran, Iran, in 2016. A total of 60 patients were included in the study, all of whom had given informed consent. Before and after the cystoscopy and two weeks later, urine analyses and urine cultures were performed. None of the patients had used antibiotics before inclusion in the study. Results: Of 60 patients (mean age: 62.67 years), 42 (70\%) were male and $18(30 \%)$ were female. The urine analysis two weeks after cystoscopy revealed eight cases of bacteriuria, of which six were bacteriuria and two were infected. There was no statistically significant relationship between age and gender in patients with symptomatic bacteriuria. There was a significant relationship between the duration of bladder cancer and symptomatic bacteriuria $(P<0.05)$. The prevalence of bacteriuria was $13.3 \%$ after the cystoscopy; its overall prevalence from various studies was $2-21.2 \%$. Conclusion: The presence of underlying cancer in patients with haematuria and cystoscopy is not an indicator of bacterial outgrowth in patients; it can be argued that prophylactic antibiotics are not required before cystoscopy.

\section{Is Prostate Biopsy Correct Enough for Decision-Making in Prostate Cancer Patients?} Alireza Ghadian

Baqivatallah University of Medical Sciences, Tehran, Iran. E-mail: P_Ghadian@yahoo.com

Objectives: Prostate cancer is the most epidemic cancer and the second leading cause of cancer mortality in men. The gold standard prostate cancer diagnosis is prostate biopsy; based on the results, the physician can determine a treatment approach. Methods: This study was conducted at the Urology Center, Baghiyyatollah al-Azam Military Hospital, Tehran, Iran, between March 2001 and March 2011. All patients who had undergone prostate biopsy and then radical prostatectomy were included in this study. Prostate-specific antigen (PSA) levels, a pathologic report of the prostate biopsy and radical prostatectomy records were collected and compared. Results: In 38\% of patients, the Gleason grade sum in radical prostatectomy was higher than in the prostate biopsy; this discrepancy was higher in patients with a PSA level $<10$ and more differentiated samples. Conclusion: A urology surgeon should consider the patient's condition and clinical conjectures in addition to the resulting Gleason grade of the biopsy to determine the best treatment, especially in making a decision for a radical prostatectomy surgery. Should the clinic and the patient's pathology be compatible, the surgery may be conducted if necessary. 


\section{Long-term Renal Outcome and Survival of Kidney Transplant Recipients Admitted to the Intensive Care Unit \\ *Amgad El-Agroudy, ${ }^{1}$ Asma Alqahtani, ${ }^{1}$ Balij Dandi, ${ }^{2}$ Eman Farid, ${ }^{2}$ Ali Al Aradhi ${ }^{2}$ \\ 'Arabian Gulf University, Manama, Bahrain;' 2Salmaniya Medical Complex, Ministry of Health, Manama, Bahrain. *Corresponding Author's e-mails: amgadelagroudy@hotmail.com and amgadeem@agu.edu.bh}

Objectives: This study aimed to evaluate the course and outcome of kidney transplant (KT) recipients admitted to the intensive care unit (ICU) at Salmaniya Medical Complex, Manama, Bahrain. Methods: Data of all adult renal transplant recipients who were admitted to the ICU between 1997 and 2017 that included demographic features, causes of end-stage renal disease (ESRD), causes of admission, time between transplantation, admission, ICU courses and outcome were included. Among $379 \mathrm{KTs}$ that were followed-up at the centre, 60 patients were admitted to the ICU and were categorised as either early (i.e. during the first three months; $\mathrm{n}=28$ ); intermediate (3-12 months; $\mathrm{n}=7$ ); or late (12 months and afterwards; $\mathrm{n}=25)$. Results: The rate of ICU admission was $15.9 \%$ and the mean age was 48.3 \pm 12.6 years. The main cause of admission was surgical complication (71\%) in the early group and infection in later groups (57\% and $80 \%$, respectively). Mortality on discharge was significantly higher in the late admission group (52\%; $P=0.0001)$ and the leading cause of death in all groups was sepsis (89\%). Twenty patients required ventilator support which was an independent risk factor for mortality $(P<0.05)$. There was a statistically significant decrease in the overall five-year and 10 -year patient survival $(P=0.031)$ in $\mathrm{KT}$ patients admitted to the ICU. Conclusions: The main reason for ICU admissions in the current study was infections especially in late admission. Mortality rate was relatively high and linked to the need for ventilators. Admission to the ICU is usually associated with decrease in graft and patient survival.

\section{Public Survey of Financial Incentives for Kidney Donation in Bahrain}

${ }^{*}$ Amgad El-Agroudy, ${ }_{1}^{1}$ Adel Alalwan, ${ }^{2}$ Mohamed Rajab²

'Arabian Gulf University, Manama, Bahrain; 'Salmaniya Medical Complex, Ministry of Health, Manama, Bahrain. *Corresponding Author's e-mail: amgadelagroudy@hotmail.com and amgadeem@agu.edu.bh

Objectives: With the increasing prevalence of end-stage kidney disease in Bahrain, kidney donation is of vital importance. This study aimed to assess how financial incentives influence individuals' views and decisions regarding kidney donation and to establish strategies to increase the number of kidneys for transplantation in Bahrain. Methods: A previously established questionnaire on financial incentives for living kidney donations was adapted for this study. The questionnaire assessed the public opinion in Bahrain on how kidney donation can be influenced by two different financial incentives, namely 10,000 Bahraini Dinar and life-long health insurance. The questionnaire was distributed electronically and a convenient sampling technique was used. Results: A total of 446 participants were included in this study, of which $39 \%$ were male and $61 \%$ were female. The majority (80\%) of participants believed that their chances for kidney donation would not increase by receiving financial compensation, while the remainder (20\%) believed it would increase. In general, married participants (70\%) found it a preferable development that health insurance companies offer financial compensation for kidney donation, while non-married participants (30\%) did not prefer this but did not believe this to be an adverse development $(P=$ 0.038). Furthermore, there was a positive correlation between age and preferable views towards financial incentives to increase kidney donation $(P<0.001)$. Conclusion: Although financial incentives might encourage a minority of the population to donate their kidney, the majority would not be influenced by implanting a financial incentives system for kidney donation.

\section{Retzius-Sparing Robot-Assisted Laparoscopic Radical Prostatectomy: Descriptive analyses and functional results of our first 25 cases}

*Pedro Sánchez de la Muela, Dr. Rubén Algarra, Dr. Ignacio Arance

Hospital Vithas Nuestra Señora de América, Madrid, Spain. *Corresponding Author's e-mail: rubenalgarra@hotmail.com

Objectives: This study aimed to analyse the immediate results, reproducibility and short urinary continence (UC) outcomes of Retziussparing robot-assisted laparoscopic radical prostatectomy. Methods: This retrospective study analysed the first 25 cases who underwent Retzius-sparing robot-assisted laparoscopic radical prostatectomy, according to Bocciardi's surgical technique described in 2010, from January 2018 to June 2019 at the Hospital Vithas Nuestra Señora de América, Madrid, Spain. Data were analysed in order to evaluate preliminary results. The primary outcome was UC (defined as zero pads or one security liner per day) one week after catheter removal. The five stages of the surgical technique, descriptive statistics and preliminary results were presented. Results: The follow-up median time was six months (range: 3-18 months). Prostate-specific antigen level median was $6.1 \mathrm{ng} / \mathrm{mL}$ (range: $4-14.3 \mathrm{ng} / \mathrm{mL}$ ). All procedures were performed by posterior intrafascial approach with bilateral neurovascular preservation in $84 \%$ of cases. Surgical margins were positive in $28 \%$ of patients, mostly in apex. Only one (4\%) patient required blood transfusion in the immediate postoperative period. The median hospital stay was 48 hours. The majority $(80 \%)$ of patients were continent one week after catheter removal. Of which, $80 \%$ do not require the use of any diapers or security pads. Most patients $(84 \%)$ were free of biochemical recurrence with a median of six months of follow-up. Conclusions: Preliminary functional results in terms of early UC were optimistic for patient's treated with Retzius-sparing robot-assisted laparoscopic prostatectomy without a worsening of the prognosis. However, these results require long-term validation.

\section{The Organisation of Medical and Diagnostic Aids for Patients with Parasitic Defeat of Urinary System \\ *Vladimir Startsev, ${ }^{1}$ Faik Asfandiyarov, ${ }^{2}$ Anton Kolmakov ${ }^{2}$ \\ 'S.M. Kirov Military Medical Academy, St. Petersburg, Russia; '2Astrakhan State Medical University, Astrakhan, Russia. *Corresponding Author's e-mail: doc.urolog@gmail.com}

Objectives: This study aimed to improve the diagnostic and treatment approach in the provision of medical care for patients with bilharzial-associated squamous cell carcinoma of the bladder (shBC). Methods: This survey-based study was conducted at medical institutions in Benguela, Angola, between 2007 and 2012. A total of 56 patients with gross haematuria who had been treated were included. Group A ( $n=35)$ included patients before applying the standardised diagnostic algorithm. Group B ( $\mathrm{n}=21)$ included patients using the new organisational format. Results: The frequency of verification of shBC in a locally advanced stage at patients of group A had been higher compared to patients in group B. Conclusions: The combined usage of cytological research of urine, ultrasonography 
and cystoscopy at the expressed proliferative process caused by USh allowed the detection of shBC in early stages on the condition of the patient's concentration in inpatient replacing structures of specialised medical institutions.

\section{The Role of Open Surgery in Children with Urolithiasis: Single centre experience *Beata Jurkiewicz and Joanna Samotyjek Department of Pediatric Surgery and Urology Center of Postgraduate Medical Education, Warsaw, Poland. *Corresponding Author's e-mail: beatajurkiewicz@wp.pl}

Objectives: In developed countries, the incidence of urolithiasis increases by $6-10 \%$ per year and its occurrence affects patients of all ages. In Poland, it is estimated that this disease affects approximately 15,000 children and about $20 \%$ of ill children require surgical treatment. The occurrence of urolithiasis in young children is usually associated with metabolic disorders or other diseases and includes X-linked diseases such as, Dent disease, Lowe syndrome and autosomal recessive syndromes (i.e. Bartter syndrome). These diseases cause the most severe forms of urolithiasis-staghorn urolithiasis. Although minimally invasive procedures such as ureteroscopic lithotripsy, retrograde intrarenal surgery, percutaneous nephrolithotomy and extracorporeal shock wave lithotripsy are commonly performed, there may still be indications for open surgery. In 2009, a combination of open and endoscopic surgery to remove kidney stones was developed at the Department of Pediatric Surgery and Urology Center of Postgraduate Medical Education, Warsaw, Poland. This procedure allows the parenchyma to remain undamaged. Methods: This study was conducted at the Department of Pediatric Surgery and Urology Center of Postgraduate Medical Education from 2016 to 2018 and included 735 children with urolithiasis who were treated. Results: During this time, a total of 33 (4.5\%) open surgeries were performed. In 20 patients, the main indication for surgery were urinary system defects with accompanying stones. Simultaneous correction of defects and removal of stones was performed. In 13 cases (1.8\%) the only indication was staghorn lithiasis with stones filling the whole calyx and pelvis system. Conclusion: If a large mass of stones is found in a small child, simultaneous pyelolithotomy with endoscopic removal of stones should always be considered, assuming that during one procedure all stones can be removed. Performing several minimally invasive procedures exposes the child to the possibility of complications that may ultimately lead to kidney failure.

\section{Percutaneous Nephrolithotripsy in Children}

*Mohammad Reza Darabi Mahboub, Mohammad Aslzare, Ameneh Ghods Department of Urology, Faculty of Medicine, Mashhad University of Medical Sciences, Mashhad, Iran. *Corresponding Author's e-mail: darabimmr@mums.ac.ir

Objectives: The formation of urinary tract stones in children commonly occurs in the upper urinary tract and requires interventional procedures such as percutaneous nephrolithotripsy (PCNL), extracorporeal shock wave lithotripsy (ESWL) and/or open nephrolithotomy. This study utilised the mini-perc technique using an instrument with only 11F compared with 24-34F sized instruments. Methods: This study was conducted from March 2003 to June 2018 on 38 children aged 18 months to 15 years who had undergone PCNL to treat urinary tract stones. Complete blood count, blood urea nitrogen, creatinine blood test, fasting blood sugar, urinary albumin and urinary creatinine were tested in all patients in addition to sonography and intravenous urography to determine kidney function and anatomical status. All patients underwent general anaesthesia during surgery. Under fluoroscopic guidance, a 30\% concentration dye was injected through the catheter and a percutaneous nephrostomy needle was inserted into the kidney and a guide wire was placed. The tract was dilated to $28 \mathrm{~F}$ and then a $28 \mathrm{~F}$ Amplatz sheet was placed and nephroscopy was done. If the patient was stone-free and haemorrhage and pelvic rupture didn't occur, tubeless PCNL was done. Results: Stones were found in the right side of 23 patients and in the left side of 15 patients. In 21 patients the stone was located in the renal pelvis, six in the inferior calyx and 11 in both pelvis and calyx. In 12 patients ESWL was done before PCNL which failed in nine patients. In three patients, the stones were broken but the particles could not be extracted. A total of 33 patients (86.8\%) were stone-free and the remaining five patients had remaining particles sized less than $6 \mathrm{~mm}$. No major complications developed, despite urinary tract infection and fever in seven patients and non-significant haematuria in 10 patients which were all cured by medical therapy. Conclusions: PCNL is more effective with less complications than ESWL and open surgery for treatment of urolithiasis in children. In patients that ESWL has not been indicated or failed, PCNL is the treatment of choice.

\section{Challenging Lymphatic Collections in Urology: Report of three cases and review of literature}

*Wadha Alqahtani and Mohammed Gomha

King Fahd Specialist Hospital, Dammam, Saudi Arabia. *Corresponding Author's e-mail: wadhaqht90@gmail.com

Large lymphatic collections can be challenging as they rarely respond completely to short-term sclerotherapies and usually need marsupilisation. We report two cases of challenging postoperative lymphoceles and one case of renal lymphangiectasia. The first case was a 51-year-old male patient who presented one month after radical prostatectomy with bilateral lower limb deep vein thrombosis and abdominal pain. Computed tomography $(\mathrm{CT})$ scan revealed huge retroperitoneal lymphocele collection, resulting in a mass effect on the urinary bladder and rectum. He was managed by ultrasonography-guided percutaneous tube drainage and povidone iodine sclerotherapy that resulted in partial response. Subsequently, he was treated by bleomycin sclerotherapy once weekly for 21 days. At the 15-month follow-up, he was asymptomatic with only $40 \mathrm{~mL}$ residual. The second case was a 27 -year-old male patient, who developed right thigh numbness on the $10^{\text {th }}$ day post-retroperitoneal lymph node dissection. CT scan revealed small paracaval collection compressing the lumen of the inferior vena cava by more than $50 \%$. He was treated by CT-guided aspiration. Follow-up CT after five weeks showed complete resolution of the collection. The third case was a 58-year-old male patient with multiple comorbidities who was referred for a large right subcapsular renal collection. CT and ultrasound findings were concordant with the diagnosis of bilateral renal lymphangiectasia that was initially drained by a percutaneous tube. Two months later, it recurred and was treated by percutaneous drainage followed by povidone iodine sclerotherapy with good response at seven years follow-up. Percutaneous drainage with sclerotherapy of long duration can be helpful in the treatment of significant lymphatic collections; this is especially important in patients who have medical concerns. Small postoperative lymphocele causing significant compression of the vena cava can be treated by aspiration by an experienced interventional radiologist. 


\section{Trans-Catheter Arterial Embolisation of the Vesical Arteries for Intractable Haematuria from Advanced Bladder and Prostate Cancers \\ *Ameer Alarayedh and Sharif Alarayedh \\ Salmaniya Medical Complex, Manama, Bahrain. *Corresponding Author's e-mail: ameer.alarayedh@gmail.com}

Objectives: Certain subsets of patients with bladder and prostate cancer have advanced disease and often develop intractable haematuria. Conservative measures frequently fail and the available treatment modalities are too aggressive for those frail patients. Minimally invasive therapeutic approaches such as trans-catheter arterial embolisation (TAE) of the internal iliac artery were first described many decades ago, however only a limited number of patients are reported in the literature. Methods: This retrospective case series was conducted at Salmaniya Medical Complex, Manama, Bahrain. All patients with intractable haematuria that had TAE from 2014 to 2019 were included. Demographic, clinical and radiographic data was collected through the patient's medical records. Data were analysed using a student's t-test. Results: Seven patients, one female and six males, with an average age of 57.2 years (range: 51-79 years) were included. Super-selective TAE of the vesical arteries with polyvinyl alcohol particles (PVA) was performed. Following embolisation, the haematuria stopped after an average of 2.6 days. There was a recurrence in haematuria in three patients; the average time to recurrence was 4.5 months. There was a significant reduction in transfusion requirements from 7.3 to 5 units of packed red blood cells, pre-and post-embolisation, respectively $(\mathrm{t}(5)=2.9,95 \%$ confidence interval $[\mathrm{CI}]=0.27-4.4 ; P=0.034$ ). A significant rise in the admission haemoglobin level from 7.8 units to 10.6 units one month post-embolisation was also noted $(\mathrm{t}(6)=4.9,95 \% \mathrm{CI}=$ 1.4-4.2; $P=0.003)$. Only one patient died due to their disease. Conclusion: TAE is an effective and safe procedure for controlling acute intractable haematuria. It should be the first-line palliative treatment of choice for patients with inoperable intractable haematuria. Embolisation of SVA with PVA particles has shown sustained control of haemorrhage with minimal side effects.

\section{Standardisation of Extended Pelvic Lymph Node Dissection at Robot-Assisted Radical Cystectomy \\ *Marat Urmantsev, Valentin Pavlov, Ruslan Safiullin, Anton Deneyko \\ Bashkkort State Medical University, Ufa, Russia. *Corresponding Author's e-mail: urmantsev85@mail.ru}

Objectives: This study aimed to review the rationale for extended pelvic lymph node dissection (ePLND) in the treatment of bladder cancer and compare robot-assisted (RA) and open ePLND. A step-by-step guide of RA ePLND was provided to illustrate the techniques currently employed at Bashkkort State Medical University, Ufa, Russia. Methods: PubMed ${ }^{\circledR}$ (National Library of Medicine, Bethesda, Maryland, USA) electronic databases were searched for English language articles on bladder cancer, ePLND and RA ePLND. The stages of radical RA cystectomy (RARC) were not standardised. Accordingly, there was no standardisation of PLND, however, ePLND improved both oncological and functional results of bladder cancer treatment. A step-by-step video and pictures with narration and accompanying explanations of each step to illustrate the authors' current techniques is available. Results: ePLND with a minimum nodal yield of 10-14 is tantamount in the treatment of bladder cancer. The number of lymph nodes resected influenced survival in both pathologically node positive and negative patients. In addition, RA ePLND was found to have equipoise as open ePLND by several groups. Conclusions: Operations should be performed in accordance with the standard extended pelvic lymph node dissection which allows for the 10-14 nodal yield mandated by the Bladder Cancer Collaborative Group and allows to safely pass the ureter under the colon using RARC and thus reduce the complications rate.

\section{Pelvic-Ureteric Junction Obstruction and Hypertension: A case report \\ *Adin Chopade, Kiran Gowdru, Allamaprabhu Sahukar, Mohan Keshavmurthy, Prem K. Krishnappa S.S. Institute of Medical Sciences and Research Centre, Davangere, Karnataka, India. *Corresponding Author's e-mail: adinbchopade@gmail.com}

Hydronephrosis is a condition involving the dilatation of the renal pelvis which is fairly common in children. Less is known about the influence of hydronephrosis on blood pressure; hypertensive effects of hydronephrosis have been suggested in experimental studies and clinical case reports. We report a six-year-old girl who presented with a mass per abdomen. Ultrasonograhy showed right gross hydronephrosis. Computed tomography-intravenous pyelogram revealed a right-sided gross hydronephrosis due to pelvic ureteric junction (PUJ) obstruction. A diethylenetriamine pentaacetic acid scan showed a grossly hydronephrotic right kidney with thinned out parenchyma and poor function $(<5 \%)$ likely due to the PUJ obstruction. Her blood pressure was $150 / 100 \mathrm{mmHg}$. Hypertension was managed by dual anti-hypertensive medications and she underwent right nephrectomy. On follow-up, the patient required single antihypertensive medication for five months postoperatively. Subsequently, her blood pressure was 100/70 mmHg and she did not require any anti-hypertensive medication. Proposed factors included vasoconstriction and salt and water overload, mediated by processes including tubuloglomerular feedback and the renin-angiotensin-aldosterone axis. Some authors describe resolution of hypertension following removal of the affected kidney, while others show that relief of the obstruction may also lead to normalisation of blood pressure. Therefore, it appears that the intrarenal mechanism leading to hypertension is reversible.

\section{Urinary Stone Composition in Mountain Areas with High Incidence of Uric Acid Stone \\ *Jalal Al-Shareef, AbdulAziz Al-Malki, Mohammad Aboelmagd \\ Department of Urology, Alhada Armed Forces Hospital, Taif, Saudi Arabia. *Corresponding Author's e-mail: Jalal0789@yahoo.com}

Objectives: Urolithiasis is a common urologic disease worldwide. The structure of the stone provides information to understand the stone formation aetiology and thereby in determining the treatment process and prevention of recurrences. This study presented the stone profile of mountain areas in Saudi Arabia and aimed to investigate the composition of urinary tract stones in patients who live in mountain areas. Methods: The study was conducted by Alhada Military Hospital, Taif, Saudi Arabia, between January 2013 and November 2018. A total of 380 urinary tract calculi were collected from 130 patients $(240$ calculi from males and 140 calculi from females). The calculi were examined and analysed to determine their chemical composition by computed infrared spectrophotometer. Results: Calcium oxalate was the most common form of stone which accounted for 34 stones, while calcium phosphate accounted for 18 stones. There were 30 uric acid stones. Calcium oxalate mixed with other components accounted for 11 stones. There were eight magnesium ammonium phosphate stones; only one cystine stone was found. The mean age was 35 years (range: $30-60$ years) and the 
male-to-female ratio was 2.9:1. Conclusion: Calcium oxalate was the most frequently encountered stone type in mountain areas; this is also the same worldwide. Uric acid stones showed a relatively higher prevalence than what is reported in the literature. The study is significant in presenting the stone profile of mountain areas in Saudi Arabia and needs further studies for evaluation and confirmation of the current findings.

\section{Leydig Cell Tumour in Elderly Patient with Presentation of Hydrocele Aneel Kumar Bhatia \\ Kettering General Hospital, Kettering, United Kingdom. E-mail: anee__kumar2000@hotmail.com}

Although hydrocele of the scrotum is relatively common, Leydig cell tumours (LCTs) are less common. The majority of LCTs are benign but malignant cases have been reported. Some cases are identified incidentally but may present with testicular changes or with features of hormone imbalance such as gynaecomastia. We report an 80-year-old sexually active non-Caucasian male patient who presented to the urology clinic with a two-year history of right testicular swelling. Clinical examination was unremarkable except from scrotal examination which showed atrophy of the left testicle and swelling of the right testicle with a positive trans-illumination test compatible with hydrocele. Ultrasound of the testes showed a large right hydrocele $28 \mathrm{~mm}$ in diameter. No focal abnormality of the right testicle or epididymis could be visualised and there was no varicocele. The patient was followed-up with repeated outpatient ultrasound scans. Two years after the initial diagnosis of hydrocele, he complained of new discomfort within the right testicle. Preoperative tumour marker serology (i.e. lactate dehydrogenase, human chorionic gonadotropin and alpha fetoprotein) was negative. However, during elective hydrocele repair, a testicular tumour was identified involving the hydrocele cavity and right radical orchidectomy was performed. The orchidectomy specimen included a disrupted, multinodular, friable tumour with a light tan cut surface. The tumour had replaced the entire testis and macroscopically involved the paratesticular tissue, hydrocele wall and spermatic cord. On microscopic examination there were typical morphological and immunohistochemical features of Leydig cell tumour. However, there were also widespread features associated with aggressive tumour behaviour. The pathological features were considered to be those of a malignant Leydig cell tumour. This is an unusual case of unexpected malignant Leydig cell tumour identified during hydrocele repair.

\section{Association Between Risk Factors and Complications in Patients Undergoing Open Radical Cystectomy \\ *Nadeem Ahmed and Abdul Hafeez Qureshi \\ The Indus Hospital, Karachi, Pakistan. *Corresponding Author's e-mail: drnadeem2012@gmail.com}

Objectives: Bladder cancer is the most frequently occurring malignancy of the urogenital system. The gold standard treatment for local control of muscle invasive bladder cancer is radical cystectomy with pelvic lymph nodes dissection. There are multiple risk factors associated with complications following open radical cystectomy. The possible risk factors that increase the rate of complications are associated with patients demographic data and comorbidities related to surgery and total length of stay in hospital, etc. This study aimed to find a positive correlation between risk factors and complications so as to improve patient care, safety and outcomes in patients undergoing open radical cystectomy. Methods: This retrospective study was conducted at the Indus Hospital, Karachi, Pakistan, from July 2016 to December 2018 and included all adult patients who underwent open radical cystectomy for bladder carcinoma. Medical profiles were assessed and different variables were noted. In addition, operative findings, number of transfusions, lymphnodes status, hospital stay and complications were taken into account. Results: A total of 36 patients (31 male and 5 females) were enrolled in the study. Patients with preoperative serum albumin of $\leq 3.5$ had 7.8 times higher odds of postoperative complications than patients who had a serum albumin of $>3.5$. Also, patients with chronic kidney disease were 5.9 times more likely to have postoperative complications than those who had no chronic kidney disease. However, results showed no significant difference in gender, comorbidities, smoking status, duration of surgery, number of transfusions and length of stay in the intensive care unit between the patients with postoperative complications and those who had no complications. Conclusion: There are multiple risk factors associated with postoperative complications. Low serum albumin and chronic kidney disease are associated with postoperative patient morbidity.

\section{Early Outcome of the Multiple Renal Arteries in Transplant Patient \\ Shailesh M. Raina}

Department of Urology, Jaslok Hospital and Research Centre, Mumbai, India. E-mail: drshaileshraina@gmail.com and drshailraina@hotmail.com

Objectives: Over the past three decades, graft and patient outcomes in kidney transplantation have improved dramatically. This study aimed to review early outcomes of transplantation using allograft(s) with multiple renal arteries in transplant patients to elucidate the usefulness of these grafts in order to improve donor pools, which was previously consider to be a relative contraindication. Methods: This prospective, observational study was conducted at the Urology Department in Jaslok Hospital, Mumbai, India, from March 2018 to April 2019. All patients who underwent renal transplant for end-stage renal disease with more than one renal arteries were enrolled in the study. All patients had a routine investigation, colour Doppler and renal scan during the early postoperative period with serum creatinine tests done every day for five days. Colour Doppler on postoperative day 5 and renal scan were done wherever indicated. Results: The incidence of complications for more than one artery transplant did not show a significant difference. Patients were followed-up for a period of three years. Conclusions: Kidneys with multiple arteries can be successfully transplanted. Accurate radiologic imaging is imperative to avoid unexpected complications during the procedure. Anastomotic technique can be modified according to the situation.

\section{The Place of Laparoscopy in Urinary Stones Management Armamentarium: Review of current trends and reporting our experience}

Mohammed Babakri

Faculty of Medicine, University of Aden, Aden, Yemen. E-mail: drbabakrigmail.com

Objectives: Surgical management of urinary stones has witnessed a major development in the last few decades. The urologist's armamentarium for treating stones became versatile by adoption of rapidly evolving technologies that increasingly replaced traditional open surgery. The introduction of laparoscopic surgery in urology practice has lagged behind other specialties, but recently there is a trend towards using laparoscopic surgery in treating various benign and malignant urological diseases. There are special situations when 
shock wave lithotripsy and endourology is not the optimal choice; in these cases, laparoscopy may replace open surgery for dealing with these cases where endourology has major limitations. The possibility of treating other associated pathologies such as ureteropelvic junction obstruction is an additional advantage of laparoscopic pyelolithotomy. This study highlighted the current international trend in laparoscopic surgery for urolithiasis and demonstrated the investigator's experience in laparoscopic stone surgery. Methods: This study was conducted from March 2011 to April 2019 in Aden, Yemen. A total of 14 consecutive patients aged 4-60 years (mean: 39 \pm 17 years) with renal and ureteral stones who underwent laparoscopic removal of their stones were included. Results: The largest diameter of a stone ranged from 25-45 mm (mean: $28 \mathrm{~mm}$ ), operative time ranged from one to four hours (mean: two hours) and hospital stay ranged from four to 10 days (mean: 5.4 days). The approach was transperitoneal in 11 patients and retroperitoneal in three patients; no major intraoperative complications occurred and no blood transfusions were needed. One patient developed prolonged urine leakage for 10 days and was managed conservatively. Follow-up after three, six and 12 months with plain abdominal X-ray, ultrasonography and urography, when indicated, showed no residual stones and no newly developed hydronephrosis. Conclusion: Laparoscopic surgery is safe and effective in management of large renal and ureter stones in patients who are not suitable candidates for endourology.

\section{Renal Parenchymal Foreign Body and a Unique Method of Removal \\ Abdul Azeez Ambalathuveettili,2}

${ }^{1}$ Baby Memorial Hospital, Kozhikode, India; ${ }^{2}$ Al Noor Polyclinic, Deira, United Arab Emirates. E-mail: drabdulazeez@roychallyassociates.com

With the advent of endourological procedures, a foreign body (FB) left in a urinary tract is not uncommon and may include forgotten stents and sometimes, broken parts of instruments or guide wire. Forceful removal of a stuck nephrostomy tube may result in breakage and may leave parts behind. Mostly, the FB is in the lumen of the collecting system and removal is straight forward. We report a 16-yearold male patient who had undergone a nephrostomy six and a half months prior and presented with occasional mild urine leakage from the nephrostomy site. Evaluation found that the patient had the distal part of the malecot catheter lodged in the kidney that had initially been used as drainage for the kidney. Percutaneous removal of the catheter was planned. However, during the procedure, it was discovered that the distal part of the tube was buried in the parenchyma with cut limbs of the "flower" of the malecot catheter protruding into the collecting system. Pulling by the limb with grasping forceps failed to dislodge it from the parenchyma. The attempt to remove the overlying tissue with biopsy forceps also failed. Therefore, a resectoscope was used to incise the overlying tissue to deliver the FB out from the parenchyma. The patient had an uneventful recovery and was doing well with sterile urine on follow-up. It was unclear how the tube got into the parenchyma, but it was probably not pushed far enough inside so that the "flower" was partly in the parenchyma. This might have produced a tissue reaction and resulted in it getting buried. Special care should be taken to place the tube properly in the desired area to prevent such an occurence.

\section{Oral Presentations}

\section{Effect of Melatonin on Nicotine Induced Fertility Changes in Wistar Albino Male Rats} Balaji Ommurugan

Kasturba Medical College, Manipal, India.E-mail: puntermmc@gmail.com

Objectives: Nicotine molecules have toxic effects on testicular functions via reactive oxygen species. Recent studies have shown that melatonin inhibits the activity of nitric oxide (NO) synthase, in addition to NO's and peroxynitrite's scavenging activity. This study aimed to evaluate the use of melatonin on reversing male fertility changes produced by nicotine. Methods: A total of 36 adult male albino rats weighing 150-200 g, aged 10-12 weeks were used in this study. The rats were divided into six groups of six rats each. Group 1 was treated with normal saline, groups 2 and 3 were administered different dosages of nicotine $(0.5 \mathrm{mg} / \mathrm{kg}$ and $1 \mathrm{mg} / \mathrm{kg}$, respectively), group 4 was given only melatonin $(6 \mathrm{mg} / \mathrm{kg} /$ day $)$, group 5 was given a combination of nicotine and melatonin $(0.5 \mathrm{mg} / \mathrm{kg}$ and $6 \mathrm{mg} / \mathrm{kg} /$ day, respecitvely) and group 6 was administered melatonin and a higher dosage of nicotine $(6 \mathrm{mg} / \mathrm{kg} /$ day and $1 \mathrm{mg} / \mathrm{kg}$, respectively). All chemicals were given orally for 30 days. Subsequently, the rats were culled. Body weight, testicular weight, testicular index, epididymis weight, sperm count and motility and viability were estimated. Histopathology of testis was done. Blood and tissue antioxidant levels were also measured. Results: Group 4 showed statistically significant maintenance of testicular functions when compared to all the other groups. Group 2 and 3 showed significant reduction in testicular function and oxidative stress when compared to all other groups. Group 5 and 6 showed significant effect on reversing the testicular toxicity and oxidative induced by nicotine and showed comparable results with the normal control rats. There was no difference between groups 5 and 6 . The histopathology of groups 1, 4, 5 and 6 showed normal testicular architecture; group 2 and 3 showed loss of germ cells with degeneration of seminiferous tubules. Conclusion: Further clinical trials should be undertaken to analyse the efficacy of melatonin as a potential agent in the treatment of infertility.

\section{Evaluation of the Beneficial Effect of Two Varieties of Coconut Water on Methotrexate-Induced Testicular Toxicity in Male Albino Wistar Rats \\ Vanishree Rao \\ Manipal College of Pharmaceutical Sciences, Manipal, India. -mail: vanim276@gmail.com}

Objectives: Methotrexate has toxic effects on testicular functions due to increased production of reactive oxygen species. Recent studies have shown that coconut water contains L-arginine and possesses antioxidant properties. This study aimed to evaluate and compare green with yellow coconut water on reversing methotrexate-induced testicular toxicity. Methods: Adult male albino Wistar rats weighing 100-200 g, aged 10 weeks old were used in the present study. A total of 30 rats were divided into five groups of six rats each. Group 1 was treated with normal saline, group 2 was given methotrexate $(20 \mathrm{mg} / \mathrm{kg})$, group 3 was administered a combination of methotrexate and green coconut water $(4 \mathrm{~mL} / 100 \mathrm{~g})$, group 4 was given methotrexate and yellow coconut water $(2 \mathrm{~mL} / 100 \mathrm{~g})$ and group 5 was given a combination of methotrexate and a higher dosage of yellow coconut water $(4 \mathrm{~mL} / 100 \mathrm{~g})$. The coconut waters were given orally for 15 days and the methotrexate was only given on day one. After the rats were culled, their body weight, testicular weight, testicular index, epididymis weight, sperm count and motility and viability were estimated. Testicular alkaline phosphatase level and serum testosterone measurement were done, in addition to histopathology of testis. Tissue antioxidant levels were also measured and analysed using one-way analysis of variance followed by post-hoc analysis. Results: Both the yellow and green coconut water-treated groups showed statistically significant maintenance of testicular functions compared to the methotrexate only treated group. Yellow 
coconut water was better than green coconut water with significant improvement in all parameters. The higher dose of yellow coconut water showed significant results compared to the lower dose. The histopathology of groups 1, 4, 5 and 6 showed normal testicular architecture; group 2 showed loss of germ cells with degeneration of seminiferous tubules. Conclusion: Green and yellow coconut water can be used as antioxidant agents in reversing testicular toxicity induced by methotrexate in rats.

\section{Sacral Neuromodulation and Saudi patients: Does it really work? \\ Mai Banakhar \\ King Abdulaziz University Hospital, Jeddah, Saudi Arabia.E-mail: drmaibanakher@hotmail.com}

Objectives: Sacral neuromodulation (SNM) is a Food and Drug Administration approved therapy for refractory overactive bladder, non-obstructive retention and frequency urgency syndrome. It is newly introduced and used in Saudi patients. This study aimed to assess satisfaction among Saudi patients who underwent SNM implantation. Methods: This cross-sectional study was conducted at the Urology Department, King Abdulaziz University Hospital, Jeddah, Saudi Arabia, and included patients who had undergone SNM implantation. A questionnaire was used to collect data regarding the patients' demographics, satisfaction level and need for combined therapy for symptom control. Results: A total of 32 patients completed the questionnaire (response rate: 58\%). Of these patients, 18 (56\%) were diagnosed with overactive bladder, seven (22\%) with chronic non-obstructive retention and seven (22\%) with pelvic pain syndrome. The median duration of therapy was two years. A total of 22 patients (69\%) received SNM therapy alone and 10 (31\%) needed additional forms of therapy for symptom control. The satisfaction level was $90 \%$; more than half the patients (20) were very satisfied and nine were moderately satisfied, while three patients were unsatisfied. Satisfaction correlated with the need for combined therapy for symptom control (correlation coefficient $=0.341 ; P=0.004$ ) but not with age, duration of therapy or complications. Conclusion: SNM therapy is associated with high patient satisfaction and is gaining acceptance amongst the Saudi population.

\section{Evaluation of the Efficacy and Safety of Hyaluronic Acid Filler in Penile Augmentation Introduction \\ Abbas Khadra \\ Clemanceau Medical Centre, Beirut, Lebanon; Dr K-Men's Health Clinic, Beirut, Lebanon. E-mail: abbas.khadra@cmc.com.lb}

Objectives: Currently, men are being offered fat as penile augmentation material but this has a high number of complications and patient dissatisfaction. Studies have shown that hyaluronic acid can be injected into the glans penis to treat premature ejaculation. Physicians are using such fillers for the penile shaft to increase its girth and thus its overall size. This study aimed to evaluate the efficacy and safety of hyaluronic acid injections into the penis as a procedure for penile enlargement. Methods: A total of 10 men were enrolled in the current study and their consent was obtained. Penile injections were given one month pre- and 12 months post-procedure in addition to a patient satisfaction questionnaire. A penile block was carried out using $10 \mathrm{~mL}$ lidocaine (2\%). Then, $10 \mathrm{~mL}$ of a filler containing highly cross-linked hyaluronic acid (2.3\%) was injected subdermally via a $25 \mathrm{G}$ cannula in the penile shaft. The filler was distributed uniformly all the way around except the area of the urethra and dorsal vascular complex. No sexual activity was recommended for one week. Results: The average patient age was 34 years. All gained a $2 \mathrm{~cm}$ increase in penile girth and $80 \%$ reported a subjective increase in penile length and boost in self-confidence and self-image. The majority (70\%) felt it improved their sexual performance and that of their wives. Almost all patients (90\%) would do the procedure again and recommend it to their friends. Results were maintained at 12 -month follow-up. Conclusion: Male genital augmentation is becoming a popular procedure. The use of fillers for such a procedure makes it attractive as it is done under local anaesthesia in the physician's office. Their use has been established to be safe in the glans penis and the current study shows that a filler containing highly cross-linked hyaluronic acid (2.3\%) is safe and effective in establishing a highly satisfactory penile augmentation result maintained at one year.

\section{Laparoscopic Partial Nephrectomy: The impact of surgeon's learning curve on the perioperative trifecta \\ *Ali Thwaini, ${ }^{1,2}$ Zeeshan Aslam² \\ 'Sulaiman Al Habib, Dubai Heathcare City, Dubai, United Arab Emirates; '2Belfast City Hospital, Belfast, United Kingdom. *Corresponding Author's e-mails: iniziaj@hotmail.com and ali.ttwaini@belfasttrust.hscni.net}

Objectives: Laparoscopic partial nephrectomy (LPN) is a highly surgically refined procedure in laparoscopic urology, with significant technical challenges. This study aimed to assess the impact of a single surgeon's learning curve on the peri-operative outcomes by analysing the trifecta of ischaemia time $<25$ minutes, negative surgical margins and no surgical complications. Methods: This retrospective study included a total of 142 consecutive patients who underwent LPN between March 2014 and March 2017. All cases were performed by a single surgeon at a tertiary centre. Patients were analysed according to their demographics and RENAL nephrometry score. The impact of the surgeon's experience on the peri-operative outcomes, as manifested by the trifecta, was analysed using multivariable regression. Outcomes for cases 42-92 (Group 1) were compared with those for cases 93-142 (Group 2). Paired t-test was used to compare the outcomes in both groups. A $P$ value $\leq 0.05$ was considered statistically significant. Results: There was no significant difference in patients' demographics nor RENAL scoring system $(P=0.09$ and 0.4 , respectively). There was no significant difference in the peri-operative blood loss $(P=0.24,95 \%$ confidence interval: $0.4-0.7)$. Warm ischaemia time was significantly less in group $2(P=0.043)$. There was no significant difference in the positive surgical margins in the two groups $(P=0.63)$. Peri-operative urine leak was significantly higher in the first group $(P<0.0001)$. Median hospital stay was similar in both groups. At three months follow-up, the renal function, determined by estimated glomerular filtration rate, was stable. No patient needed renal replacement therapy. Conclusion: LPN represents a steep learning curve. At 142 cases there seems to be an improvement in the peri-operative outcomes mainly in terms of urine leak. However, the learning curve plateau is yet to be reached at this number. More cases are needed and longer follow-up would be helpful.

\section{Tumour Bearing Kidneys as Potential Transplant Donors: A national online survey *Ali Thwaini, ${ }^{1,2}$ Zeeshan Aslam² 'Sulaiman Al Habib, Dubai Healthcare City, Dubai, United Arab Emirates; ${ }^{2 B}$ Belfast City Hospital, Belfast, United Kingdom. *Corresponding Author's e-mails: iniziaj@hotmail.com and ali.thwaini@belfasttrust.hscni. net}

Objectives: According to the UK annual kidney-transplant report in March 2018, 4,757 adult patients are on the UK kidney-transplant list, with only 3,272 transplants performed; 1,200 patients died while waiting for their transplant. More than 7,000 nephrectomy 
operations were recorded at the British Association of Urological Surgeons audit outcome for 2014; 2,900 nephrectomy procedures were performed on patients with low-stage renal cell carcinoma. This study re-explored the acceptability of utilising small tumourbearing kidneys as potential organ donors, after ex vivo tumour removal and pathology confirming benign pathology or low risk cancer with clear margins. Methods: An online survey was carried out across UK urologists with special interest in renal surgery, transplant surgeons and nephrologists, between November and December 2018. Respondents were asked whether they would consider such kidneys for transplantation in selected groups of dialysis dependent patients. Results: A total of 110 urologists, 70 nephrologists and 50 transplant surgeons were approached. Of which 60 (response rate: 55\%) urologists, 41 (response rate: 59\%) nephrologists and 28 (response rate: 56\%) transplant surgeons completed the questionnaire. The majority of urologists (72\%) and transplant surgeons (93\%) and some nephrologists (43\%) supported the concept of using these kidneys for selected transplant recipients. Conclusion: There is general acceptance for using those kidneys for transplantation in the UK. This concept has its ethical and logistical challenges, though it's been performed before. This survey should encourage further studies to address the critical unmet need of kidneys for transplantation.

\section{Percutaneous Transvesical Single-Port Robotic Simple Prostatectomy}

Guilherme Sawczyn, Rair Valero, Roger Yau, *Jihad Kaouk

Cleveland Clinic, Cleveland, USA. *Corresponding Author's e-mails: guivsaw@hotmail.com and kaoukj@ccf.org

Objectives: This study presented the investigator's initial experience performing percutaneous transvesical single-port robotic simple prostatectomy (pSP-RSP) using the novel da Vinci SP ${ }^{\circledR}$ Robotic Platform (Intuitive Surgical Inc., Sunnyvale, California, USA). Methods: Four consecutive patients were treated with pSP-RSP between February and March 2019. Using the percutaneous transvesical approach, the patient was positioned in the supine position, a $3 \mathrm{~cm}$ infra-umbilical incision was made after percutaneous needle identification of the bladder dome, a GelPOINT Mini advanced access platform (Applied Medical Resources Corporation, Rancho Santa Margarita, California, USA) was inserted directly into the bladder and the da Vinci SP ${ }^{\circledR}$ robot (Intuitive Surgical Inc.) was docked. Pneumovesicum was achieved, the prostate was accessed and the adenoma was enucleated. Finally, a mucosal advancement flap was sutured to the urethra to cover the resected area. Results: The mean operative total time was 182.2 minutes, mean estimated blood loss was $350 \mathrm{~mL}$ and the mean postoperative haemoglobin deficit was $2.5 \mathrm{mg} / \mathrm{dL}$. The mean length of stay was 37 hours and 26 minutes. Half of the patients (50\%) left the surgery with a 2-way Foley catheter and without bladder irrigation. None of the patients received surgical drains and there was no need for additional ports. No intraoperative complications were reported and no surgeries were converted to an open approach. There were no blood transfusions during or following the procedure. Conclusion: The pSP-RSP is a minimally invasive option for treating benign prostatic obstruction with substantially enlarged glands. In the current study, this approach led to minimal bleeding, true single incision robotic surgery, no bladder irrigation, minimal usage of postoperative opioids and a shorter hospital stay with the possibility of outpatient management in the future. Further studies need to be done with larger sample sizes and long-term follow-up periods to confirm these findings.

\section{Four Hours Hospital Stay Radical Prostatectomy: The Cleveland Clinic single- port experience \\ Guilherme Sawczyn, Rair Valero, Roger Yau, *Jihad Kaouk \\ Cleveland Clinic, Cleveland, USA. *Corresponding Author's e-mails: guivsaw@hotmail.com and kaoukj@cct.org}

Objectives: This study showcased the investigator's experience of outpatient single-port robotic assisted laparoscopic prostatectomy for prostate cancer with the new da Vinci SP ${ }^{\circledR}$ Robotic Platform (Intuitive Surgical Inc., Sunnyvale, California, USA). Methods: A database was compiled prospectively of the first consecutive patients who underwent an extraperitoneal prostatectomy using the da Vinci SP ${ }^{\circledR}$ Robotic Platform (Intuitive Surgical Inc.). A new practice was adopted by the investigators of sending the patients home shortly after the recovery unit like an ambulatory surgery patient. The data of all patients who underwent this surgery and the protocol were reviewed retrospectively. Results: Since implementing the da Vinci SP ${ }^{\circledR}$ Robotic Platform (Intuitive Surgical Inc.), the standard approach has been changed to a true single incision extraperitoneal approach and the patients were sent home the same day. A total of 20 consecutive patients were included in this study, of which 11 were discharged the same day of surgery. The median length of stay was 4.3 hours. The median patient reported pain scale was 2 out of 10; opioid use was only 15\%. There were no intraoperative complications. Postoperative complications included one pelvic abscess, a deep vein thrombosis and pulmonary embolism and one urine leak. One patient had a superficial seroma in the incision which was drained by opening the skin. No transfusions were administered. The positive margin rate was 5. Follow-up ranged from one week to three months. Conclusions: With the new protocol of using the da Vinci SP ${ }^{\circledR}$ Robotic Platform (Intuitive Surgical Inc.) with an extraperitoneal approach, the investigators have been able to perform a relatively painless procedure where patients can go home within four hours of the procedure with minimal opioid use.

\section{Utilising a New Robotic Surgical System to Treat Challenging Urinary Stones Mohamad Salkini West Virginia University, Morgantown, USA. E-mail: mhdsalkini@gmail.com}

Objectives: A worldwide increase in the incidence and prevalence of urolithiasis has been observed. The standard treatment of urologic stone disease (USD) has changed from open surgery to extracorporeal shock wave lithotripsy, percutaneous nephrolithotomy (PCNL) or ureteroscopy depending on the size and location of the stone. Open stone surgery is reserved for challenging urinary stones (CUS) that are not amenable to the above-mentioned techniques. The investigator used the new da Vinci $\mathrm{SP}^{\circledR}$ Robotic Platform (Intuitive Surgical Inc., Sunnyvale, California, USA) to treat CUS. This study aimed to share the investigator's experience of the da Vinci SP Robotic Platform (Intuitive Surgical Inc.) to treat patients with urolithiasis instead of an open surgical approach. Methods: This study included a total of 26 patients who underwent robotic assisted stone surgery (RSS) between January 2010 and March 2019 at West Virginia University, Morgantown, USA, for USD involving 29 nephroureteral units. Results: A total of 29 RSS were accomplished with no conversion to open surgery. Three patients had bilateral stones and needed to have RSS on each side separately. RSS were performed on the right-side in 13 surgeries. The indications for RSS included morbid obesity $\left(\mathrm{n}=8\right.$, mean body mass index: $\left.56.4 \mathrm{~kg} / \mathrm{m}^{2}\right)$, need for concurrent renal surgery $(n=8)$ severe contractures limiting positioning for retrograde endoscopic surgery or PCNL ( $\mathrm{n}$ $=4)$, symptomatic calyceal diverticular stone with failed endoscopic approach $(\mathrm{n}=4)$ and inability to position the patient $(\mathrm{n}=20)$. Most nephroureteral units (90\%) were rendered stone-free on the first attempt with complications occurring after five surgeries (17\%). Conclusion: RSS is a viable option in the treatment of CUS with a high success rate and low risk of complications. The need for open 
stone surgery was eliminated by RSS at the current centre. Multiple centres need to participate in RSS with larger numbers of patients to create an established role for RSS.

\title{
The Dissected Lymph Node Burden Based Predictive Model for Predicting Biochemical Recurrence after Radical Prostatectomy
}

Mehdi Kardoust Parizi

Department of Urology, Shariati Hospital, Tehran University f Medical Sciences, Tehran, Iran. E-mall: m.kardoust@yahoo.com

Objectives: This study aimed to assess the dissected lymph node (LN) burden-based predictive model for predicting biochemical recurrence after radical prostatectomy in patients with non-metastatic prostate cancer (Pca).Methods: This study was conducted between May 2009 and April 2018 on patients with non-metastatic Pca who underwent radical retropubic prostatectomy. Clinicopathological parameters including age, preoperative prostate-specific antigen, prostate weight at surgery, Gleason score (GS) of surgical specimen, pathological tumour stage, dissected LN number, positive LN and positive LN density were recorded. Cox regression models were used to analyse the prognostic value of assessed potential prognosticators and designing a predictive model. Results: A total of 216 consecutive patients were included and had a mean age of 63.9 years (range: $40-83$ years). Positive surgical margins were present in $16.6 \%$. Final GS was $2-6$ in $39.7 \%, 7$ in $38.3 \%$ and $8-10$ in $22 \%$. The mean number of LNs removed during pelvic lymph node dissection was 11.96 (range: $2-31$ ). Of all patients, 7.4\% were LN-positive; patients with negative LNs had significantly better progression-free survival $(P<0.05)$. Dissected LN number was associated with a lower biochemical recurrence rate in patients with final negative LNs (odds ratio: 0.971). Conclusions: Although the prognostic value of radical prostatectomy (RP) driven prognosticators such as GS and tumour stage have been frequently used to predict oncological outcomes after RP, the dissected LN burden could improve the accuracy of predictive tools. Moreover, this study confirmed that LN-negative PCa patients benefit from LN dissection in terms of oncological outcomes.

\section{Possibilities of the Use of Raman Spectroscopy in the Diagnostics of In Vitro Urinary Bladder Cancer \\ Valentin Pavlov, *Rita Gilmanova, Vyacheslav Korolev \\ Bashkir State Medical University, Ufa, Russia. *Corresponding Author's e-mail: marganitagilmanova@mail.n}

\begin{abstract}
Objectives: Bladder cancer is the most common malignant tumour of the urinary tract. Raman spectroscopy is the method that makes it possible to study tissues at a molecular level with high specificity during pathological transformation. This study aimed to evaluate the diagnostic capabilities of the Raman spectroscopy method in detecting bladder cancer. Methods: A total of 20 bladder tissue samples with a histologically confirmed diagnosis of bladder cancer and 13 samples of normal bladder tissue were examined. The research of samples used the following machine configuration: wavelength $=785 \mathrm{~nm}$, grating $=1,200 \mathrm{~g} / \mathrm{mm}$, filter $=100 \%$, confocal opening $=300$ microns and integration time $=50$ seconds. Spectral data were analysed. Results: There were significant differences in the Raman spectra from the data obtained from bladder cancer tissue and normal bladder tissue. The Raman-fluorescent spectra of bladder cancer samples contained strongly pronounced peaks in the range of $500-2,500 \mathrm{~cm}^{-1}$. It is necessary to note the high intensity of the peaks of $750 \mathrm{~cm}^{-1}$ (thymine), $1,000 \mathrm{~cm}^{-1}$ (phenylalanine), $1,100 \mathrm{~cm}^{-1}$ (a complex of fatty acids), which were absent in the spectra of samples of normal tissues. In addition, there was a significant increase in the intensity of peaks in the region of $850 \mathrm{~cm}^{-1}$ and $1,250 \mathrm{~cm}^{-1}(\mathrm{C}-\mathrm{NH} 2$ bond in adenine, guanine and cytosine molecules) in the spectra of bladder cancer, as well as the appearance of groups of low intensities in the region of 2,800-3,000 $\mathrm{cm}^{-1}$ (lipids and proteins). Conclusion: The studied bladder cancer tissue samples showed an increase in the intensity of Raman light scattering compared to samples of normal bladder tissue; this confirms the spectral differences in the biochemical composition of these samples.
\end{abstract}

\section{Is Extracorporeal Shock Wave Lithotripsy Still the Treatment of Choice for Renal and Upper Ureteric Calculi: Our experience with 300 cases and its comparison with retrograde intrarenal surgery literature}

*Kush Shah, Piyush Singhania, Aman Agarwal, Ajinkya Patil

MGM Institute of Health Sciences, Navi Mumbai, India. *Corresponding Author's e-mail: kush_dgreat@yahoo.co.in

Objectives: Extracorporeal shock wave lithotripsy (ESWL) is used in the management of renal calculi with a size greater than $2 \mathrm{~cm}$. With the recent advances in endourology, the use of ESWL has become less frequent. Newer techniques such as retrograde intrarenal surgery and percutaneous nephrolithotomy are replacing ESWL for treatment of renal stones. Methods: This study was conducted at the Department of Urology, MGM Institute of Health Sciences, Navi Mumbai, India, between October 2015 and October 2018. Patients who underwent intravenous pyelogram X-ray and had a calculi size of $6-20 \mathrm{~mm}$ were included. Patients with untreated urinary tract infection, pregnancy, musculoskeletal deformities and deranged coagulation profile were excluded. Double-J stenting was done prior to the procedure for stones $>1 \mathrm{~cm}$. A maximum of three ESWL sessions were given. The procedure was done under local anaesthesia for adults and under general anaesthesia for children. Results: A total of 300 patients between the ages of 15 months and 78 years were included in the current study. There was no effect of age and gender on stone clearance. The overall success rate was $91.97 \%$. Success rates for smaller calculi were significantly higher $(6-10 \mathrm{~mm}$ : $97.7 \%, 10-15 \mathrm{~mm}$ : $90.1 \%$ and $15-20 \mathrm{~mm}$ : $87.5 \% ; P<0.05)$. Success rates of calculi in the lower calyx (83.63\%) was less compared to calculi in the upper calyx (96.77\%), middle calyx (96.15\%), renal pelvis $(90 \%)$ and upper ureter $(88.23 ; P<0.05)$. Complications of the procedure included pain $(28$ patients required no treatment and three patients required admission and treatment), haematuria (15 patients required no treatment and two patients required admission and treatment) and one patient had perinephric gemstone treated conservatively. Conclusion: ESWL is a safe, effective procedure in treatment of renal calculi with clearance rates of up to $91.97 \%$ and can still be regarded as the treatment of choice for renal stones especially in developing countries where cost is still a major factor. 


\section{Stone Extraction Might be More Suitable than Stone Fragmentation}

*Rishikesh Pandya, Venkatesh Seetharam, Amir Ali

MMC Specially Hospital Abu Dhabi, Abu Dhabi, United Arab Emirates. *Corresponding Author's e-mails: rishikesh.pandya@nmc.ae and dmrpandya@yahoo.com

Objectives: Complex stones (i.e. large stones or single or multiple stones) frequently need more than one procedure before the stone is released. Increased hospital admissions and procedures under anaesthesia means more stress for the patient and burden on the healthcare system. Usually a percutaneous approach is used, instead the investigators propose a method to select patients where stone extraction without fragmentation might overcome the above-mentioned challenges. Methods: This study was conducted over a period of five years and included kidneys with single or multiple stones, a volume larger than $4 \mathrm{~cm}$ and more than $2 \mathrm{~cm}$ in the ureter. Based on computed tomography scan, the kidneys with more than $40 \%$ of renal pelvis lying outside the medial border of the kidney (extrarenal) were operated by conventional laparoscopic approach. Stones in anomalous kidney locations such as a ureteropelvic junction (UPJ) obstruction/ectopic kidney/crossed-fused were also included. Single sitting stone-free rates were assessed. Stones that missed extraction were termed residual stones. Results: A total of 28 cases, 19 kidneys and nine ureteric stones were analysed. Stones were extracted without fragmentation by conventional transperitoneal laparoscopic pyelotithotomy and intrarenal stones were extracted using a flexible cystoscope and basket. UPJ obstruction was found in 14 patients, five had dismembered pyeloplasty and nine had nondismembered Fenger pyeloplasty. One pelvic kidney, one crossed-fused ectopia and two horseshoe kidneys were rendered stone-free. Complete stone clearance was achieved for 21 kidney stones; two required flexible ureteroscopy. Complete clearance was achieved in all ureteric stones. After two days of antibiotics, no peritonitis or sepsis was reported. Drains were removed on postoperative day three in the morning and the patient was discharged. Conclusions: Laparoscopic extraction in selected cases with suitable anatomy allows non-fragmented complete stone clearance. The procedure is safe and suitable for kidneys with extra renal pelvis. In anomalous kidneys, laparoscopic approach might be more suitable than percutaneous.

\section{Effectiveness of Frenulum Subcutaneous Injection of Botulinum Toxins in the Treatment of Premature Ejaculation \\ Mohammed Ismail \\ College of Medicine, University of Baghdad, Baghdad, Iraq. E-mail: mohammed_allbassil@yahoo.com}

Objectives: Premature ejaculation is a common sexual disorder, which is usually underreported. Multiple treatment methodologies are in use due to the absence of an effective, universally acceptable treatment modality. This study aimed to evaluate the effectiveness of frenulum subcutaneous injection of 10 international units (IU) of botulinum toxins in patients with premature ejaculation, who were dissatisfied with classical treatment. Methods: This study included 30 patients who reported premature ejaculation who were unhappy with the classical treatment either due to its adverse effects or because of its overall inefficacy. They were treated with a frenulum subcutaneous injection of 50 IU botulinum toxins. Pre- and post-treatment intravaginal ejaculatory latency time (IELT) was analysed. Results: Before injection, the IELT ranged from one to four minutes with a mean of $1.86 \pm 1.008$ minutes. After injection, the IELT ranged from one to 45 minutes with a mean of $13.6 \pm 7.82$ minutes. The difference between IELT before and after the injection was statistically significant $(P=0.001)$. Conclusion: Frenulum subcutaneous injection of botulinum toxins in the treatment of premature ejaculation is a safe and effective way to treat premature ejaculation.

\section{Safety and Efficacy of the Novel Plugging Technique Using a Haemostatic Agent for Port-Site Closure in Minimally Invasive (Robotic and Laparoscopic) Different Urological Surgeries: Tertiary Centre Experience}

Abdulrahman Alsuwailim, ${ }^{1}$ Khalid Albalawi, ${ }^{2}$ Maher Moazin, ${ }^{3}$ Meshal Almutair, ${ }^{4}$ Abdullah Alaliyah, ${ }^{5}$ Naif A. Aldhaam ${ }^{3}$

${ }^{1}$ King Faisal University, Ahsaa, Saudi Arabia; ${ }^{2}$ Tabuk University, Tabuk, Saudi Arabia, ${ }^{3}$ King Fahad Medical City, Rivadh, Saudi Arabia; ' Q Qassim University, Qassim, Saudi Arabia, ${ }^{5}$ King Khalid University, Abha, Saudi Arabia. *Corresponding Author's e-mail: Daho0om.1@hotmail.com

Objectives: Many techniques and devices have been used to close surgical ports after minimally invasive procedures; however, it may entail several complications. Therefore, this study aimed to determine the safety and efficacy of the novel plugging technique using SURGICEL ${ }^{\circledR}$ (Ethicon Inc., Bridgewater, New Jersey, USA) to close port-sites. Methods: This retrospective study was conducted at King Fahad Medical City, Riyadh, Saudi Arabia, over an eight year period. All patients who underwent any minimally invasive urological surgery in which the surgical ports were closed using the SURGICEL ${ }^{\circledR}$ (Ethicon Inc.) plugging technique were included. Patients age, gender, type of surgery and the follow-up results were collected from their electronic medical records and analysed. The sum of each type of urological minimally invasive (robotic and laparoscopic) surgery was identified, as well as the rate of associated specific type(s) of complication. Results: A total of 113 patients, of which 54 were male with a mean age of $41.7 \pm 18.4$ years and 63 were females with a mean age of $39.7 \pm 17.9$ years, were included. The male-to-female ratio was 1:1.2. Among those patients, two cases developed portsite discharge, one case developed a port-site hernia but the majority (97.4\%) had no port-site complications. Conclusion: This study showed that the plugging technique of port-site postoperatively using a haemostatic agent is safe, easy, effective and results in a low rate of port-site complications. Surgeons should ensure that SURGICEL ${ }^{\circledR}$ (Ethicon Inc.) is neither thrown inside nor placed above the level of subcutaneous tissue to avoid the development of a hernia or misdiagnosing a discharge as an infection.

\section{Long-Term Safety and Efficiacy of Neurosacral Modulation in Patient with Hypocontractile Bladder: A series of 10 cases \\ Shailesh M. Raina \\ Department of Urology, Jaslok Hospital and Research Centre, Mumbai, India.E-mail: drshaileshraina@gmail.com and drshailraina@hotmail.com}

Objectives: This study aimed to determine the safety and efficacy of sacral neuromodulation (SNM) in patients with non-obstructive urinary retention in which the bladder was hypocontractile as demonstrated on urodynamic studies. Methods: A total of 10 patients who underwent staged neuromodulation non-obstructive urinary retention from 2007 to 2019 at Jaslok Hospital and Research Centre, Mumbai, India, were included. Patients who had a 50\% improvement in symptoms after one week of stage 1 procedure qualified for 
permanent device implantation. Those who showed restoration of spontaneous voiding with a residual volume of $<100 \mathrm{~mL}$ or who experienced a 50\% reduction in catheterisation frequency were offered permanent stage 2 SNM device insertion. Patient data were assessed for efficacy and need for intermittent self-catheterisation, complications and operative revision rates. All patients' medical history, physical examination, voiding diaries, urodynamic studies and cystoscopic examination were reviewed. Results: The majority (70\%) of patients returned to a spontaneous micturition with no need of clean intermittent catheterisation and no major complaint of frequency, urgency or postoperative infection. One patient was lost to follow-up. The device had to be removed from two patients (one for due to noncompliance and the other due to infection). Conclusion: SNM is a relatively new and safe method that can improve the quality of life of a patient who is on clean intermittent catheterisation for non-obstructive bladder dysfunction.

\section{Robotic Partial Nephrectomy for Renal Tumours: Do we really require intra-operative ultra-sonography for margin clearance? \\ Shailesh M. Raina \\ Department of Urology, Jaslok Hospital and Research Centre, Mumbai, India.E-mails: drshaileshraina@gmail.com and drshailraina@hotmail.com}

Objectives: Currently, there is a significant increase in indications and demands of nephron-sparing surgery for renal masses. Laparoscopic partial nephrectomy has a steep learning curve. This minimally invasive technique aims to accomplish precise tumour resection and renal reconstruction while minimising warm ischaemia time. Complex renal tumours may preclude a minimally invasive approach to nephron-sparing surgery in some patients. The da Vinci SP ${ }^{\circledR}$ Robotic Platform (Intuitive Surgical Inc., Sunnyvale, California, USA) is seen as especially useful in this regard. This study reports the investigator's technique of robotic partial nephrectomy for challenging renal tumours and tumour margin clearance without intraoperative ultrasonography. Methods: The study was conducted from April 2017 to February 2019 and used the da Vinci SP ${ }^{\circledR}$ Robotic Platform (Intuitive Surgical Inc.) to perform partial nephrectomies. For complex posterior- and hilar-located tumours with sequential steps of colon mobilisation and hilar dissection, only the renal artery was clamped. In cases of multiple arteries, selective artery clamping was done. Whole kidney mobilisation and flipping anterior kidney anteriorly for posteriorly located tumours was done. Tumour scoring before excision was done intraoperatively and no intraoperative ultrasonography was used. The tumours were removed and single-layer renorrhaphy was used. Results: Robotic partial nephrectomy procedures were performed successfully without complications. A total of 45 robotic partial nephrectomies were done, with an average warm ischaemia time of 17 minutes and an average intraoperative blood loss was $150 \mathrm{~mL}$. Histopathology confirmed renal cell carcinoma with clear margins in all cases. The hospital stay was 3-4 days. At the three-month follow-up, no patients experienced perioperative complications. The one-year follow-up computed tomography scan showed no recurrence. Conclusions: Robotic assistance may facilitate tumour resection and renal reconstruction for challenging cases, with complete margin clearance achieved without intraoperative ultrasonography, offering a minimally invasive surgical option and no difference with or without the use of intraoperative ultrasound in margin clearance. Intraoperative ultrasound may only be required in very complex cases such as cystic (papillary) carcinoma.

\section{Superficial Circumflex lliac Artery Perforator Flap Phalloplasty Could be a Standard of Care in Gender Reassignment Surgery for the Future \\ Sanjay Pandey}

Kokilaben Dhinubhai Ambani Hospital \& Research Institute, Mumbai, India. E-mail: saniaypdr@gmail.com

Objectives: Gender reaffirmation phalloplasty is a complex surgical task. Successful construction of the penis must meet certain cosmetic and functional thresholds. The ideal neophallus should be sensate, hairless and similar in colour to the surrounding skin. In addition, the surgical scars should be inconspicuous, maintain rigidity for sexual intercourse and allow for micturition upon standing with minimum donor site morbidity. This report demonstrated the investigator's technique of using a superficial circumflex iliac artery perforator (SCIP) flap for phalloplasty and its evolution over 19 cases. Methods: After appropriate psychiatric and endocrine evaluation, patients were counselled for surgery. Concomitant mastectomies/vaginectomies were also done when feasible. The SCIP phalloplasty was the first part of a three stage process that involved urethroplasty and insertion of a penile prosthesis. Results: A total of 19 cases were reviewed in the current study. The mean age of the patients was 28 years (range: 21-39 years). There were no complications in 14 cases, but there was failure in three patients where the flap had to be debrided as the vascularity was hampered because of a narrow vessel, obesity or pedicle stretch-twist. Two of them had prostheses-related issues during the neourethra construction. Conclusions: The free radial forearm flap, though it established itself over time, has major problems such as donor-site morbidity with large depressive scarring after skin grafting, urethral fistulas and need for microvascular anastomosis. A SCIP flap has the advantage of minimal donorsite morbidity with a concealed donor scar and no microvascular anastomosis. It facilitates a stepwise approach towards complete establishment of the neophallic state.

\section{Urinary Undiversion for the Young and Needy: A stitch in time \\ Sanjay Pandey \\ Kokilaben Dhirubhai Ambani Hospital \& Research Institute, Mumbai, India. E-mail: sanjaypdr@gmail.com}

Undiversion implies a change in patient management after judicious re-evaluation and the decision to offer the patient the possibility of re-establishing continuity of the urinary tract once a permanent form of urinary diversion had been chosen in the past. Careful consideration is given to the historical reason for the permanent diversion. The current status of the patient should be judged on various parameters including the patient's psychology and the possible consequences if the results of undiversion are less than optimal. The author reports a 26-year-old male patient who was subjected to a radical cystectomy with an ileal conduit seven years prior. The ileal conduit was taken down and incorporated as a part of the neobladder from ileal segments that were created in the patient. He had an intact sphincter mechanism zone that was evaluated by cystoscopy preoperatively. His postoperative recovery was assisted with CISC programmed regimen. At one-year follow-up he was voiding well to near-completion (plateau shaped flow prolonged flow pattern) with insignificant post-void residue. A postoperative cystoscopy showed a capacious bladder with a few mucous plugs. 